\title{
ON NUMBER OF EXCITON SURFACE STATES IN MULTILAYER MOLECULAR SLABS
}

\author{
NGUYEN BA AN* \\ International Center for Theoretical Physics \\ P.O. Box 586, 34100 Trieste, Italy
}

(Received July 21, 1997; in final form September 25, 1997)

\begin{abstract}
Criteria for the possible number of exciton surface states in a multilayer molecular slab are elucidated in detail. Namely, we explicitly determine the domains of system parameters where the size dependence plays a role. We further clarify the way a surface state is localized in the slab in both asymmetric and symmetric configurations. Basing on our results which are obtained within the first nearest layer approximation, we also conjecture properties to be expected when further nearest layers are taken into consideration.
\end{abstract}

PACS numbers: 71.35.Aa, 73.20.At, 73.20.Dx

\section{Introduction}

Practical optoelectronic devices are based on characteristics of both light and material. For the material, semiconductor low-dimensional crystals have been widely used in which the Wannier-Mott excitons are the key figures giving rise to fast responses and room-temperature operations [1]. Recently, by novel techniques of organic molecular beam deposition, organic molecular superlattices [2] and periodic assemblies of quantum dots [3] have successfully been produced. This has motivated the study of the physics and the possible applications of heterostructure-based molecular crystals in which the electronic transitions are mainly due to the Frenkel excitons (see, e.g., [4-6] and references therein). Theoretical investigations of unique properties of future composite organic-inorganic quantum structures, where the Wannier-Mott and the Frenkel excitons hybridize, have just been started [7-9].

One type of the available heterostructure-based molecular systems is multilayer molecular slabs which have currently attracted a lot of interest [10-12]. Reference [10] evaluated the superradiance rate neglecting interaction between the layers. In Refs. [11] and [12] the authors calculated the internal field and the

*Permanent address: National Center for Natural Sciences and Technology, Institute of Physics, P.O. Box 429 Bo Ho, Hanoi 10000, Vietnam. 
third-order susceptibility taking into account the interlayer coupling but ignoring distortion of the wave function near the interfaces separating the slab region from the regions of the surrounding materials. Since the primarily necessary knowledge for any calculation of the optical responses is the knowledge about the material system, a thorough study of the electronic structure of the material system deserves its own significance. If simple unrealistic "no-escape" boundary conditions like in Refs. [11] and [12] are imposed, there arises only one kind of eigenstates, the propagating states (or the bulk states). In reality, due to finite size, each layer feels different sets of neighbors and different distances from the two surrounding materials which, in turn, may have different dielectric properties when compared to each other as well as to the slab material. An accurate account of the real boundary conditions at the slab interfaces is therefore necessary. Such kind of work has been done, e.g., in [13] in which the possible number of exciton surface states (from now on we just call surface states instead of exciton surface states for short) is shown to depend not only on the conditions at the slab interfaces but also on the slab thickness. In this work we go into more detail in the sense that we explicitly indicate the domains of system parameters where the size dependence is important. Outside those domains the surface state number is determined solely by the boundary conditions, not by the thickness. When a surface state exists, a question is to which of the two interfaces is it localized? And, when there appears more than one surface state, what is the degree of localization of each of these states? Answering these questions is also a part of the present work.

\section{Size dependence versus surface-to-bulk ratios}

The Hamiltonian of an $N$-layer molecular slab can be written in terms of coupled in-layer excitons as (Here we consider $N \geq 3$. The special cases of $N=1,2$ when all layers are surface ones were studied in [14].)

$$
H=\sum_{n}\left[E_{n} b_{n}^{+} b_{n}+\sum_{n^{\prime} \neq n} R_{n, n^{\prime}} b_{n^{\prime}}^{+} b_{n}\right],
$$

where $n, n^{\prime}$ run from 1 to $N, b_{n}\left(b_{n}^{+}\right)$are the operators for the $n$-th layer exciton with energy $E_{n}$. (Note that $E_{n}$ differ from layer to layer and are not equal to each other as oversimplified in Refs. [11] and [12]. This feature is essential in giving rise to the appearance of surface states.) $R_{n, n^{\prime}}$ measures the interaction between layers $n$ and $n^{\prime}$. Both $E_{n}$ and $R_{n n^{\prime}}$ can be calculated taking into account both the dipolar interaction by a planewise method $[15,6]$ and the abrupt change of media at the interfaces by the method of image charges [16]. Obviously, $E_{n}$ and $R_{n n^{\prime}}$ are determined by properties of the whole system: the slab plus the surrounding materials. We shall refer to them as the system parameters. Because the interlayer interaction holds for all the layers together in the slab, it does not seem a good approximation to fully neglect it as was done in Ref. [10]. Nevertheless, as a well-known fact $[15,6], R_{n, n^{\prime}}$ falls off very quickly against $\left|n-n^{\prime}\right|$ so that one can limit oneself to the interaction within a few numbers of nearest layers only. If the 1st (2nd, 3rd, $\ldots, L$-th $(L \ll N))$ nearest layers are taken into consideration, the approximation is called the 1 st $(2 \mathrm{nd}, 3 \mathrm{rd}, \ldots, L$-th) nearest layer approximation and abbreviated as 1NLA (2NLA, 3NLA; ..., LNLA). 
In the $L N L A$ there are $2 L$ outermost special layers with different energies $E_{1}, E_{2}, \ldots, E_{L}, E_{N-L}, E_{N-L+1}, \ldots, E_{N-1}, E_{N}$ and $N-2 L$ internal layers with equal energy $E=E_{L+1}=E_{L+2}=\ldots=E_{N-L-2}=E_{N-L-1}$. The $N$ eigenfunctions $\Psi_{\nu}$ and $N$ eigenenergies $\omega_{\nu}(\nu=1,2, \ldots, N)$ satisfying the Schrödinger equation

$$
H \Psi_{\nu}=\omega_{\nu} \Psi_{\nu}
$$

can be determined by representing $\Psi_{\nu}$ as

$$
\Psi_{\nu}=\sum_{n} g_{n}^{\nu} b_{n}^{+}|0\rangle
$$

which is justified by the fact that $\left|R_{n n^{\prime}}\right|$ are much smaller than $E_{n}$ in most realistic systems. The coefficients $g_{n}^{\nu}$ and $\omega_{\nu}$ are then to be found from $2 L+1$ difference equations which are obtained upon the substitution of Eq. (3) into (2). It can be shown that the slab eigenstates can be classified into bulk states, which hop from layer to layer throughout the whole slab region, and surface states, which are localized near some outer layers of the slab. For definiteness let us present our results using the 1NLA since in many cases the 1NLA proves to be sufficient. Later we shall discuss on the LNLA briefly.

In the 1NLA there are 3 difference equations to be solved

$$
\begin{aligned}
& \left(\omega_{\nu}-E_{1}\right) g_{1}^{\nu}=R g_{2}^{\nu} \text {, } \\
& \left.\begin{array}{l}
\left(\omega_{\nu}-E\right) g_{n}^{\nu}=R\left(g_{n-1}^{\nu}+g_{n+1}^{\nu}\right), \quad 1<n<N, \\
\left(\omega_{\nu}-E_{N}\right) g_{N}^{\nu}=R g_{N-1}^{\nu},
\end{array}\right\}
\end{aligned}
$$

where $E=E_{2}=E_{3}=\ldots=E_{N-1}$ and $R=R_{n, n^{\prime}=n \pm 1}$. The solution to Eq. (4) can be found in the form [13]

$$
\left.\begin{array}{ll}
g_{n}^{l, k_{i}}=(-1)^{n l}\left(\theta_{l, k_{i}} \mathrm{e}^{n k_{i}}+\vartheta_{l, k_{i}} \mathrm{e}^{-n k_{i}}\right) & \text { for surface states } \\
g_{n}^{q_{j}}=\alpha_{q_{j}} \cos \left(n q_{j}\right)+\beta_{q_{j}} \sin \left(n q_{j}\right) & \text { for bulk states }
\end{array}\right\}
$$

and

$$
\left.\begin{array}{ll}
\omega_{l, k_{i}}=E+(-1)^{l} 2 R \cosh k_{i} & \text { for surface states } \\
\omega_{q_{j}}=E+2 R \cos q_{j} & \text { for bulk states }
\end{array}\right\}
$$

where $\left\{k_{i}\right\}=\left\{k_{1}, k_{2}, \ldots, k_{s}\right\}, l$ equals either 1 or 2 and $\left\{q_{j}\right\}=\left\{q_{1}, q_{2}, \ldots, q_{N-s}\right\}$. Note, here and hereafter $k_{i}, q_{j}$ are treated in units of the layer spacing and thus are dimensionless. The coefficients in Eq. (5), which satisfy the normalization condition, are given by

$$
\begin{aligned}
\theta_{l, k_{i}} & =\left(C_{k_{i}}+2 B_{l, k_{i}}+B_{l, k_{i}}^{2} C_{-k_{i}}\right)^{-1 / 2}, \quad \vartheta_{l, k_{i}}=B_{l, k_{i}} \theta_{l, k_{i}}, \\
\beta_{q_{j}}= & \left\{\frac{N}{2}\left(A_{q_{j}}^{2}+1\right)+\frac{\sin \left(N q_{j}\right)}{2 \sin q_{j}}\left[\left(A_{q_{j}}^{2}-1\right) \cos \left[(N+1) q_{j}\right]\right.\right. \\
& \left.\left.+2 A_{q_{j}} \sin \left[(N+1) q_{j}\right]\right]\right\}^{-1 / 2} \\
\alpha_{q_{j}} & =A_{q_{j}} \beta_{q_{j}}
\end{aligned}
$$


with

$$
\begin{aligned}
& A_{q_{j}}=-\frac{D_{1} \sin q_{j}}{D_{1} \cos q_{j}+1}, \quad B_{l, k_{i}}=-\frac{D_{1} \mathrm{e}^{k_{i}}+(-1)^{l}}{D_{1} \mathrm{e}^{-k_{i}}+(-1)^{l}}, \\
& C_{k_{i}}=\frac{1-\mathrm{e}^{2 N k_{i}}}{\mathrm{e}^{-2 k_{i}}-1}, \quad D_{1, N}=\frac{E-E_{1, N}}{R} .
\end{aligned}
$$

As $E-E_{1, N}$ characterize the conditions at the slab interfaces while $R$ the bulk interaction, we conventionally call $\left|D_{1, N}\right|$ the surface-to-bulk ratios. Clearly, there are $s$ surface states and $N-s$ bulk states. The value of $l$ and $s$ values of $k_{i}$ are to be determined from the transcendental equation

$$
\mathrm{e}^{2 N k_{i}}=\frac{D_{1} D_{N} \mathrm{e}^{2 k_{i}}+(-1)^{l}\left(D_{1}+D_{N}\right) \mathrm{e}^{k_{i}}+1}{\mathrm{e}^{2 k_{i}}+(-1)^{l}\left(D_{1}+D_{N}\right) \mathrm{e}^{k_{i}}+D_{1} D_{N}}
$$

while the $N-s$ values of $q_{j}$ from

$$
\sin q_{j} \cot \left(N q_{j}\right)=\frac{D_{1}+D_{N}+\left(D_{1} D_{N}+1\right) \cos q_{j}}{D_{1} D_{N}-1} .
$$

Analyzing Eqs. (7) and (8) leads to the result that $s$ may be 0,1 or 2 depending on the relation between $D_{1}, D_{N}$ and $N$. The criteria for the possible number of surface states are as follows [13]:

$$
\left.\begin{array}{ll}
t_{M} \equiv \frac{N\left|D_{1}+D_{N}\right|+\sqrt{G}}{2(N+1)} \leq 1, & s=0 \\
t_{m} \equiv \frac{|N| D_{1}+D_{N}|-\sqrt{G}|}{2(N+1)} \leq 1<t_{M}, & s=1 \\
1<t_{m}, & s=2
\end{array}\right\}
$$

with

$$
G=\left(N^{2}-1\right)\left(D_{1}-D_{N}\right)^{2}+\left(D_{1}+D_{N}\right)^{2} .
$$

The noticeable message coming from Eq. (9) is that the number of surface states depends not on $D_{1}$ and $D_{N}$ separately but rather on their combinations $\left|D_{1}+D_{N}\right|$ and $\left|D_{1}-D_{N}\right|$ expressing the physical fact that the two interfaces are related or, in other words, they are interacted or correlated. And, moreover, there is a dependence on the slab thickness through $N$. The size dependence is significant especially in mesoscopic slabs because the number of surface states as well as other electronic properties could be tailor-made by changing the slab thickness but keeping the same surrounding media. From Eq. (9) one may think that the size dependence always goes together with the dependence on the surface-to-bulk ratios. However, we shall prove that the size dependence plays its role only in certain domains of the system parameters. Outside those domains there are no size dependences at all. Let us formulate this by a sequence of statements. i.e.,

Statement 1: If both the surface-to-bulk ratios are less than or equal to 1 ,

$$
\left|D_{1}\right| \leq 1 \text { and }\left|D_{N}\right| \leq 1
$$

then there are always no surface states for an arbitrary slab thickness.

Statement 2: If one of the surface-to-bulk ratios is less than or equal to 1 and the other is greater than 2, i.e.,

$$
\left|D_{1(N)}\right| \leq 1 \text { and }\left|D_{N(1)}\right|>2
$$


then there is always one surface state for an arbitrary slab thickness.

Statement 3: If both the surface-to-bulk ratios are greater than 2, i.e.,

$\left|D_{1}\right|>2$ and $\left|D_{N}\right|>2$,

then there are always two surface states for an arbitrary slab thickness.

Statement 4: If one of the surface-to-bulk ratios is less than or equal to 1 and the other is greater than 1 but less than or equal to 2, i.e.,

$$
\left|D_{1(N)}\right| \leq 1 \text { and } 1<\left|D_{N(1)}\right| \leq 2 \text {, }
$$

then there may be zero or one surface state depending on the slab thickness.

Statement 5: If one of the surface-to-bulk ratios is greater than 2 and the other is greater than 1 but less than or equal to 2 , i.e.,

$$
\left|D_{1(N)}\right|>2 \text { and } 1<\left|D_{N(1)}\right| \leq 2 \text {, }
$$

then there may be one or two surface states depending on the slab thickness.

Statement 6: If both the surface-to-bulk ratios are greater than 1 but less than or equal to 2 , i.e.,

$$
1<\left|D_{1}\right| \leq 2 \text { and } 1<\left|D_{N}\right| \leq 2,
$$

then there may be one or two surface states depending on the slab thickness.

Clearly, from the above statements, there is a fixed number of surface states independent of $N$ in the domains of the surface-to-bulk ratios which lie outside the interval between 1 and 2 (Statements 1,2 and 3). Only when one (as in Statements 4,5 ) or both (as in Statement 6 ) of the ratios fall within that interval, the size dependence shows up. The proofs of Statements 1 to 3 are given in Appendix. Here illustrations are made for Statements 4 to 6 . For Statement 4 let us choose $\left|D_{1}\right|=0.4$ and $\left|D_{N}\right|=1.05$ (1.1). The numerical calculation shows that there exists a critical $N_{\mathrm{c}}=19(9)$ such that for $N \leq N_{\mathrm{c}}$ there are no surface states whereas for $N>N_{\mathrm{c}}$ there is one surface state. The change of the surface state number from one to zero when $N$ crosses $N_{\mathrm{c}}$ while decreasing is physically understood as follows. For large $N\left(N>N_{\mathrm{c}}\right)$ the two interfaces, being far from each other, are almost independent. However, for small $N\left(N \leq N_{\mathrm{c}}\right)$ the interfaces are relatively close to each other and become correlated. Their correlation results in the suppression of the surface state which exists for large $N$. Statement 5 is elucidated by choosing $\left|D_{1}\right|=2.1$ and $\left|D_{N}\right|=1.1$ (1.2). A critical thickness appears to be $N_{\mathrm{c}}=12(7)$. Now for $N \leq N_{\mathrm{c}}$ there is one surface state but for $N>N_{\mathrm{c}}$ the surface state number is two. The crossover at $N=N_{\mathrm{c}}$ of the surface state number from two to one when $N$ decreases is also explained by the interfaces correlation in thin slabs which in this circumstance suppresses one of the two surface states present in thick slabs. Finally, the size dependence underlying Statement 6 is qualitatively similar to that in Statement 5 . The critical $N_{\mathrm{c}}=13(11)$, say, for $\left|D_{1}\right|=1.1$ and $\left|D_{N}\right|=1.5(4.5)$. Note that the $N$-governed change of the surface state number satisfies a "selection rule": $\Delta s=0, \pm 1$ because $t_{m}<t_{M}$ under any situation. Statements 1 to 6 presented above are general and, thus, contain as a particular case the result of Ref. [17] where. it was possible to analytically express $N_{\mathrm{c}}$ as a function of $|D|=\left|D_{1}\right|=\left|D_{N}\right|$. Only Statements 1, 3 and 6 are needed for the symmetric configuration used to draw Fig. 2 in [17]. 


\section{Ways in which surface states are localized}

Having understood the possible surface state number which is determined by the surface-to-bulk ratios and size of the slab, we are now going to clarify the way of localization of surface states. For this purpose we study the spatial profile $\left|g_{n}^{l, k}\right|^{2}$ given in (5) for a surface state. First we consider the case of two surface states. Take $\left|D_{1}\right|=2.5$ and $\left|D_{N}\right|=10$. In accordance with Statement 3 there exist, for such parameters, two surface states for any $N$. Let $N=20$ and solve Eq. (7) for $\left(l, k_{i}\right)$. The solutions are $s_{1}=\left(l_{1}, k_{1}\right)=(1,-0.9163)$ for the first surface state and $s_{2}=\left(l_{2}, k_{2}\right)=(1,-2.3026)$ for the second. The spatial profile is now ready to be plotted. Figure 1 shows $\left|g_{n}^{l_{1}, k_{1}}\right|^{2}$, which are big circles connected by

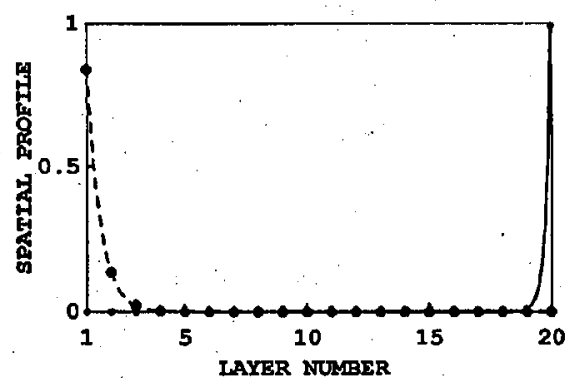

Fig. 1. Spatial profiles of surface states, i.e., $\left|g_{n}^{l_{i}, k_{i}}\right|^{2}$ as functions of $n$ for $N=20$, $\left|D_{1}\right|=2.5$ and $\left|D_{20}\right|=10$. There are two surface states. The one at the right interface is stronger localized since $\left|D_{20}\right|=10>\left|D_{1}\right|=2.5$.

a dashed curve, and $\left|g_{n}^{l_{2}, k_{2}}\right|^{2}$, which are small circles connected by a solid curve, as functions of $n$. Transparently, each surface state is separately localized near an interface: $\left|g_{n}^{l_{i}, k_{i}}\right|^{2}$ are finite at $n=1$ and $n=20$ but fall off exponentially in the bulk. However, the localization degree differs at $n=1$ and $n=N$. From Fig. 1 it follows that the degree of localization is stronger (weaker) near the interface whose surface-to-bulk ratio is greater (smaller). If the surrounding material on the left is replaced by another one that makes, say, $\left|D_{1}\right| \leq 1$ (or $1<\left|D_{1}\right| \leq 2$ ), then according to Statement 2 (Statement 5 ) one surface state always exists for any $N$ (may exist for $N \leq N_{\mathrm{c}}$ ). Numerical calculations show that when there is only one surface state this state is always localized near the interface with a greater surface-to-bulk ratio (see Fig. 2 and its caption). A question can be asked at this point: to which interface is localized the only one existing surface state when both the surface-to-bulk ratios are equal like in a symmetric configuration? When $|D|=\left|D_{1}\right|=\left|D_{N}\right|$ the only one surface state may exist within the domain $1<|D| \leq 2$ provided that $N \leq N_{\mathrm{c}}=(|D|+1) /(|D|-1)$. Taking $|D|=1.4$ we get $N_{\mathrm{c}}=6$. Thus, for $N=5<6$ the only possible surface state has the characteristics $s=(l, k)=(1,-2.3026)$. Its spatial profile is sketched in Fig. 3 . For $N=10>6$ (with the same $|D|=1.4$ ) the number of surface states becomes two: one has the characteristics $s_{1}=\left(l_{1}, k_{1}\right)=(1,-0.3565)$ and the other has $s_{2}=\left(l_{2}, k_{2}\right)=(1,-0.3058)$. The ways these two surface states are localized are 


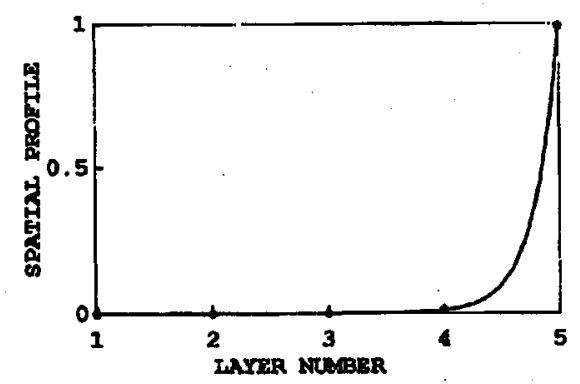

Fig. 2. The same as in Fig. 1 but for $\left|D_{1}\right|=1.2$ and $N=5$. In this situation there is a size dependence having $N_{\mathrm{c}}=6$. For $N=5<N_{\mathrm{c}}$ the only existing surface state is localized near the right interface since $\left|D_{5}\right|=10>\left|D_{1}\right|=1.2$.

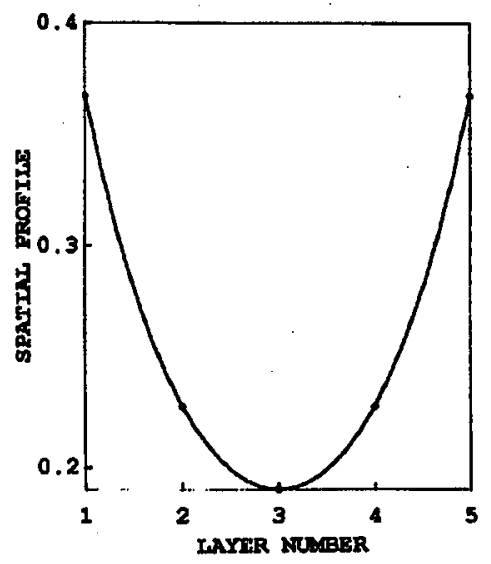

Fig. 3. The same as in Fig. 1 but for a symmetric configuration $\left|D_{1}\right|=\left|D_{N}\right|=1.4$. For $N=5<N_{c}=6$ (note the accidental coincidence of $N_{c}=6$ here and in Fig. 2) there exists only one surface state which is localized equally near each of the interfaces.

visualizable in Fig. 4 in which the circles connected by a dashed curve belong to the state $s_{1}$ and those connected by a solid curve to the state $s_{2}$. From Figs. 3 and 4 it is evident that, no matter how many surface states exist, each of them is equally localized near each of the two interfaces. That is, more explicitly speaking, a surface state is half attaching to the left interface and half to the right one. This feature appears reasonable as required by the symmetry of the configuration concerned: the coefficient $g_{n}^{l, k}$, from a symmetry point of view, should be either a symmetric or an antisymmetric function of $n$ with respect to the slab center.

\section{Conclusion and discussion on further approximations}

We have studied in detail the criteria determining the surface state number in a multilayer molecular slab. The possible number of surface states depends on the combination of conditions at both the interfaces which are in general corre- 


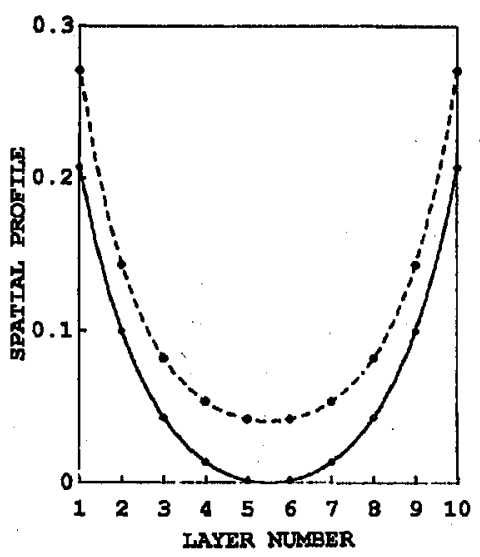

Fig. 4. The same as in Fig. 3 but for $N=10>N_{\mathrm{c}}=6$. There arise two surface states each of which is localized equally near each of the interfaces.

lated with each other. In small size slabs this interaction may lead to the size dependence which is manifested in certain domains of the system parameters. If the surface-to-bulk ratios are both too small $(\leq 1)$ or one is too small but the other large enough $(>2)$ or both large enough, then the number of surface states is fixed to be zero or one or two, respectively, whatever is the slab thickness. Only when one or both of the surface-to-bulk ratios is/are not too small and not large enough, the size dependence plays its role. In this case, there is a critical thickness $N_{\mathrm{c}}$ and the surface state number is reduced by one for $N \leq N_{c}$ as compared to that for $N>N_{\mathrm{c}}$. It is worth emphasizing that, as a consequence of the size dependence versus the surface-to-bulk ratios, $N_{\mathrm{c}}$ is subject to the system parameters. That is, slabs having the same thickness may possess different surface state numbers for different conditions at the interfaces.

Naturally, the size dependence is of paramount importance in mesoscopic heterostructures which are of great interest nowadays. In macroscopically sized systems each interface contributes separately because their correlation is negligible. This is easily obtained from our criteria in the limit $N \gg 1$ in which Eq. (9) reduces to

$$
\left.\begin{array}{ll}
t_{M} \rightarrow\left|D_{M}\right| \leq 1, & s=0 \\
t_{m} \rightarrow\left|D_{m}\right| \leq 1<\left|D_{M}\right|, & s=1 \\
1<\left|D_{m}\right|, & s=2
\end{array}\right\}
$$

where $\left|D_{m}\right|=\min \left\{\left|D_{1}\right|,\left|D_{N}\right|\right\}$ and $\left|D_{M}\right|=\max \left\{\left|D_{1}\right|,\left|D_{N}\right|\right\}$. We realize that simply $\left|D_{m}\right|$ and $\left|D_{M}\right|$ enter Eq. (17), not their combinations $\left|D_{m} \pm D_{M}\right|$ as in Eq. (9), reflecting the physical fact that in macroscopic slabs the interaction between the two interfaces is negligible and plays no role in determining the number of surface states. In particular, if $\left|D_{m}\right|=\left|D_{M}\right|$, i.e. $\left|D_{1}\right|=\left|D_{N}\right|$, as in the symmetric configuration, Eq. (17) recovers the result of Ref. [18]. Our criteria (9) are therefore general: they apply to any size and boundary conditions. The salient merit of 
Eq. (9) is their validation in mesoscopic slabs like currently interested molecular quantum wells consisting of from several to some tens layers in total.

In asymmetric slabs, if there are two surface states, each of them is localized near an interface and the degree of localization is stronger at the interface which has greater surface-to-bulk ratio. If only one surface state arises, it is localized near the interface whose surface-to-bulk ratio is larger. In symmetric slabs the way of localization differs. Surface states, if any, are always localized equally at each of the two interfaces.

As to further approximations we could conjecture the following: a possible number of surface states in an $N$-layer molecular slab within the $L$ NLA may be $0,1,2, \ldots, 2 L-1$ and $2 L$ depending both on combinations of the surface-to-bulk ratios and on the total number of layers. Nevertheless, general analytical criteria revealing dependences on both material parameter combinations and size are immensely difficult to obtain. A recent work [19] has developed the 2NLA for the same structure but restricted to the symmetric configuration of large size only. For such a simplified model no size dependence and no possibilities of one and three surface states could be derived although, as compared to the 1NLA, four surface states and an additional type of surface state that evanesces as an exponent multiplied by an oscillating amplitude have been pointed out in Ref. [19]. Understanding the nature and the number of surface states as well as the relevant size dependence in the finite size multilayer molecular slab is still a matter of intensive research which is really actual since there have been available experiments [20] waiting for theoretical explanations.

\section{Acknowledgments}

This work was financially supported by SAREC, UNESCO and IAEA during the stay of the author at the International Center for Theoretical Physics, Trieste, Italy. The hospitality of ICTP and its Condensed Matter Section is gratefully acknowledged.

\section{Appendix}

- This appendix proves Statements 1,2 and 3. All the proofs will go with the assumption $\left|D_{1}\right| \geq\left|D_{N}\right|$ (the inverse situation $\left|D_{N}\right| \geq\left|D_{1}\right|$ should proceed along the same lines with the exchange $1 \leftrightarrow N$ to be made). Let $\phi=\operatorname{sgn}\left(D_{1} D_{N}\right)= \pm 1$ be the sign of the product $D_{1} D_{N}$. Explicitly, in terms of $\phi$, the quantities defined in Eqs. (9) and (10) read

$$
\begin{aligned}
& G(\phi)=\left(N^{2}-1\right)\left(\left|D_{1}\right|-\phi\left|D_{N}\right|\right)^{2}+\left(\left|D_{1}\right|+\phi\left|D_{N}\right|\right)^{2}, \\
& t_{M}(\phi)=\frac{N\left(\left|D_{1}\right|+\phi\left|D_{N}\right|\right)+\sqrt{G(\phi)}}{2(N+1)} \\
& t_{m}(\phi)=\frac{\phi\left[N\left(\left|D_{1}\right|+\phi\left|D_{N}\right|\right)-\sqrt{G(\phi)}\right]}{2(N+1)}
\end{aligned}
$$

\section{Define}

$$
H_{ \pm}(\phi)=\left[N\left(\left|D_{1}\right|+\phi\left|D_{N}\right|\right) \pm 2(N+1)\right]^{2} .
$$




\section{Proof of Statement 1}

A little algebra yields

$$
G(\phi)-H_{-}(\phi)=4(N+1)\left[N\left(\left|D_{1}\right|-1\right)\left(1-\phi\left|D_{N}\right|\right)+\left(\phi\left|D_{1} D_{N}\right|-1\right)\right] .
$$

The rhs has been grouped so that it is obviously non-positive by virtue of (11). Then

$$
\sqrt{G(\phi)} \leq\left|N\left(\left|D_{1}\right|+\phi\left|D_{N}\right|\right)-2(N+1)\right|=2(N+1)-N\left(\left|D_{1}\right|+\phi\left|D_{N}\right|\right) .
$$

The latter equality is again due to (11). We thus have

$$
t_{M}(\phi) \leq 1
$$

that implies no surface states at all. Statement 1 has been proved.

\section{Proof of Statement 2}

The same lhs can be manipulated into another form for the rhs, namely

$$
\begin{aligned}
& G(\phi)-H_{-}(\phi) \\
& =4(N+1)\left\{\left(1-\phi\left|D_{N}\right|\right)\left[(N-2)\left(\left|D_{1}\right|-1\right)+\left(\left|D_{1}\right|-2\right)\right]+\left(\left|D_{1}\right|-1\right)\right\} .
\end{aligned}
$$

The rhs has now been grouped in another way as compared to the grouping in the Proof of Statement 1 so that it is obviously positive by virtue of (12). Then

$$
\sqrt{G(\phi)}> \pm\left[2(N+1)-N\left(\left|D_{1}+\phi\right| D_{N}\right)\right] \text {. }
$$

The upper "plus" gives

$$
t_{M}(\phi)>1
$$

while the lower "minus" gives

$$
t_{m}(1)<1 \text {. }
$$

Now

$$
G(-1)-H_{+}(-1)=-4(N+1)\left[N\left(1-\left|D_{N}\right|\right)\left(\left|D_{1}\right|+1\right)+\left|D_{1} D_{N}\right|+1\right]
$$

is obviously negative as seen from its grouping when (12) is used. Then

$$
\sqrt{G(-1)}<\left|N\left(\left|D_{1}\right|-\left|D_{N}\right|\right)+2(N+1)\right|=N\left(\left|D_{1}\right|-\left|D_{N}\right|\right)+2(N+1) .
$$

The latter equality is due to the assumption $\left|D_{1}\right| \geq\left|D_{N}\right|$. The inequality is nothing else but

$$
t_{m}(-1)<1
$$

We thus have for both $\phi= \pm 1$

$$
t_{m}(\phi)<1<t_{M}(\phi)
$$

that implies one surface state. Statement 2 has been proved. 


\section{Proof of Statement 3}

When Eqs. (13) are satisfied, algebraic manipulations yield the following groupings:

$$
\begin{aligned}
& H_{-}(1)-G(1)=4(N+1)\left[(N-3)\left(\left|D_{1}\right|-1\right)\left(\left|D_{N}\right|-1\right)\right. \\
& \left.\quad+2\left(\left|D_{N}\right|-1\right)\left(\left|D_{N}\right|-2\right)+\left(\left|D_{1}\right|-\left|D_{N}\right|\right)\left(2\left|D_{N}\right|-3\right)\right]>0
\end{aligned}
$$

that lead to

$$
t_{m}(1)>1 \text {, }
$$

and

$$
\begin{gathered}
G(-1)-H_{+}(-1)=4(N+1)\left[(N-3)\left(\left|D_{1}\right|+1\right)\left(\left|D_{N}\right|-1\right)\right. \\
\left.+2\left(D_{N}^{2}-2\right)+\left(\left|D_{1}\right|-\left|D_{N}\right|\right)\left(2\left|D_{N}\right|-3\right)\right]>0
\end{gathered}
$$

that lead to

$$
t_{m}(-1)>1 \text {. }
$$

We thus have for both $\phi= \pm 1$

$$
t_{m}(\phi)>1
$$

that implies two surface states. Statement 3 is proved.

As seen from the above proofs or, in general, from the criteria (9) containing only $\left|D_{1} \pm D_{N}\right|$, we note that the number of surface states is unaffected by the relative signs of $D_{1}$ and $D_{N}$. Note, however, that the energy level of a surface state is sign-sensitive $[13,19]$.

\section{References}

[1] E. Hanamura, Opt. Quantum Electron. 21, 441 (1989).

[2] F.F. So, S.R. Forrest, Y.Q. Shi, W.H. Steier, Appl. Phys. Lett. 56, 674 (1990); D.-Y. Zang, Y.Q. Shi, F.F. So, S.R. Forrest, W.H. Steier, Appl. Phys. Lett. 58, 562 (1991); F.F. So, S.R. Forrest, Phys. Rev. Lett. 66, 2649 (1991).

[3] V.L. Alivisatos; M.C. Schiamp, P. Alivisatos, Nature 370, 354 (1994).

[4] L. Belleguie, S. Mukamel, Phys. Rev. B 52, 1963 (1995); N. Wang, V. Chernyak, S. Mukamel, J. Chem. Phys. 100, 2465 (1994); N. Wang, S. Mukamel, Chem. Phys. Lett. 231, 373 (1994); F. Spano, S. Mukamel, Phys. Rev. Lett. 66, 1197 (1991).

[5] T. Tokihiro, Y. Manabe, E. Hanamura, Phys. Rev. B 47, 2019 (1993); ibid. 51, 7655 (1995).

[6] Nguyen Ba An, E. Hanamura, Phys. Lett. A 199, 249 (1995).

[7] V.M. Agranovich, R. Atanasov, F. Bassani, Solid State Commun. 92, 295 (1994).

[8] V.I. Yudson, P. Reineker, V.M. Agranovich, Phys. Rev. B 52, R5543 (1995).

[9] Nguyen Ba An, Phys. Lett. A 214, 99 (1996).

[10] V.M. Agranovich, O.A. Dubovsky, K.I. Grigorishin, T.A. Leskova, P. Reineker, Chem. Phys. 203, 11 (1996).

[11] H. Ishihara, K. Cho, in: Proc. Int. Symp. on Science and Technology of Mesoscopic Structures, Nara 1991, Eds. S. Namba, C. Hamaguchi, T. Ando, Springer-Verlag, Berlin 1992, p. 464. 
[12] H. Ishihara, K. Cho, Phys. Rev. B 48, 7960 (1993).

[13] Nguyen Ba An, E. Hanamura, J. Phys., Condens. Matter 8, 2273 (1996).

[14] M.R. Philpott, J. Chem. Phys. 61, 5306 (1974); M.R. Philpott, P.G. Sherman, Phys. Rev. B 12, 5381 (1975).

[15] M.R. Philpott, J. Chem. Phys. 58, 588 (1973).

[16] E. Hanamura, N. Nagaosa, M. Kumagai, T. Takagahara, Mater. Sci. Eng. B 1, 225 (1988).

[17] Nguyen Ba An, E. Hanamura, Mod. Phys. Lett. B 9, 1609 (1995).

[18] V.I. Sugakov, Sov. Phys.-Solid State 5, 1607 (1964); 14, 1711 (1973).

[19] Nguyen Trung Dan, E. Hanamura, Phys. Rev. B 54, 2739 (1996).

[20] Ya. Aaviksoo, Ya. Lippamaa, T. Reinot, Opt. Spektrosk. 62, 706 (1987). 